\title{
Modelización y monitorización de procesos de refusión láser de recubrimientos depositados por plasma
}

\author{
J. M. AMAdO, C. ÁlVAREZ, G. NICOLÁS, A. J. LÓPEZ, J. A. PÉREZ, A. RAMIL, E. SAAVEDRA, J. SANESTEBAN, M. J. TOBAR, A. YÁÑ̃EZ \\ Departamento de Ingeniería Industrial II. Universidad de La Coruña. Ferrol.
}

\begin{abstract}
Este artículo describe un estudio teórico y experimental del proceso de refusión, por láser de $\mathrm{CO}_{2}$, de recubrimientos depositados por plasma. Se trata de lograr mediante el proceso de refusión una buena unión metalúrgica entre recubrimiento y sustrato, entre las diferentes partículas que componen el recubrimiento y de este modo evitar que el recubrimiento sea frágil y tenga una baja adherencia con el sustrato. En una primera aproximación la modelización sirvió de ayuda para lograr los parámetros adecuados que permitiesen fundir toda la capa proyectada junto con una mínima porción del sustrato para minimizar el grado de dilución. La modelización presentada incluye algunos aspectos que ayudan a describir el cambio de fase asociado a la refusión, estos son la utilización de las propiedades termofísicas adecuadas y la variación del coeficiente de absorción en el paso de la fase sólida a la fundida. Los resultados de la monitorización refuerzan el conocimiento del proceso y ayudan a la validación del modelo. Los resultados consisten en la predicción, con bastante precisión, de la forma y tamaño de las regiones fundidas para diferentes condiciones del proceso, lo que permite realizar el estudio paramétrico con la ayuda del computador, minimizando pruebas experimentales.
\end{abstract}

Palabras Clave: Refusión por láser, Modelo de elementos finitos, Transmisión de Calor, Monitorización en Tiempo Real.

\section{Modelling and monitoring of laser refusion processes of coatings obtained by plasma}

This paper shows a theoretical and experimental study of the $\mathrm{CO} 2$ laser remelting of plasma sprayed coatings. The aim of the process is the decrease of the porosity to improve the layer properties. In a first approach simulation helps in finding the right process parameters, those parameters allow the melting of the sprayed layer and a minimal amount of substrate material to minimize dilution. The presented modelling includes several features allowing the description of phase transition in the remelting process, the main point is the use of different absorption coefficient in the two phases. Monitoring results gave additional information about the process and another way of doing process validation. The main result are is the prediction size and shape of the remelted layers and due to that the simulation code can be used in the parameter finding and plays an important role in the complete understanding of the process.

Keywords: Laser remelting, Finite Element Modelling, Heat Transfer, On-line Monitoring

\section{INTRODUCCIÓN}

Los procesos industriales de tratamiento de materiales con láser más tradicionales son el corte, la soldadura, el taladrado y los tratamientos superficiales, todos ellos han sido ya estudiados tanto de manera teórica como experimental, como denominador común a todos ellos es necesario hacer notar que los parámetros experimentales que han de ser ajustados son la potencia incidente, la velocidad relativa entre la pieza y el haz y el diámetro de este último.

Entre los tratamientos térmicos superficiales se encuentra la refusión por láser, en este caso se trata de refundir recubrimientos de una aleación tipo Ni-Cr-B-Si depositados por plasma sobre AISI 304, el objetivo es mejorar, a través de la refusión, la unión metalúrgica de las diferentes partículas que componen la capa proyectada y de éstas con el sustrato para así mejorar la unión entre el recubrimiento y el material base y disminuir la fragilidad de la capa proyectada.

En el contexto de esta modelización el haz láser se considera como una fuente de calor en movimiento con una distribución de intensidad bien conocida que induce una serie de condiciones de contorno sobre la superficie del material. Este modelo incluye efectos realistas tales como propiedades termofísicas dependientes de la temperatura (calor específico, densidad y conductividad térmica), La simulación del haz incluye la distribución espacial de su energía que es la que corresponde al modo $\mathrm{TEM}_{01}$ * La validación del modelo se realiza a través de los registros de los diferentes sensores y de la determinación de la forma de la región fundida.
En lo que sigue se presentará el modelo propuesto junto con alguno de los resultados obtenidos que sirven para la validación del mismo.

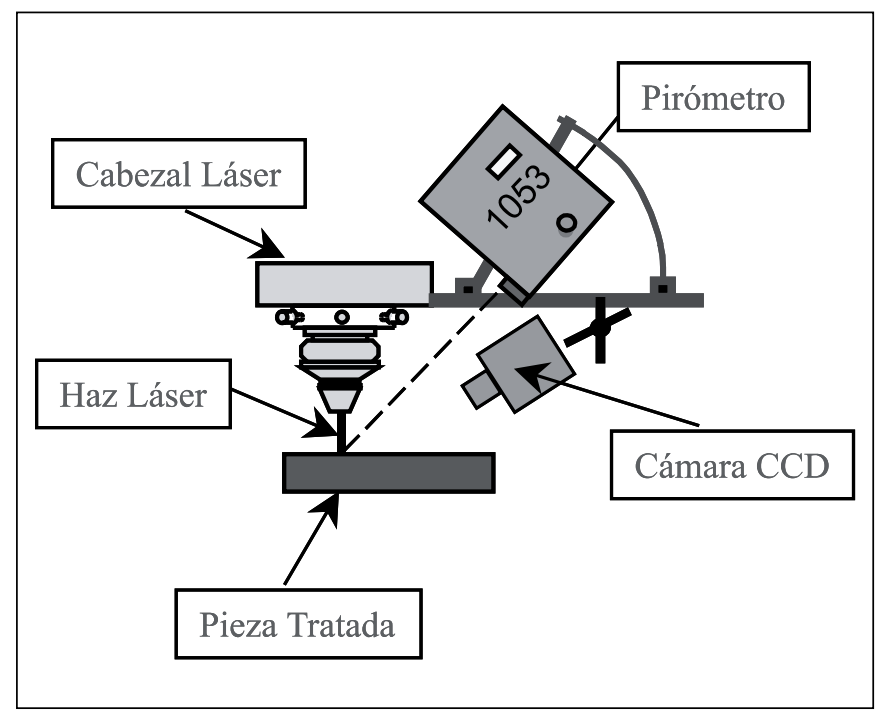

Figura 1. Representación esquemática del dispositivo experimental con el pirómetro y la cámara CCD. 


\section{Dispositivo Experimental}

Un laser de $\mathrm{CO}_{2} 2200 \mathrm{~W}$ laser se utiliza como fuente de irradiación para las piezas que van a ser tratadas. Para la lectura de la temperatura en la superficie del material se utiliza un pirómetro que cubre el rango que va desde los $723 \mathrm{~K}$ hasta $2123 \mathrm{~K}$, con una precisión mejor que el $1 \%$. En el caso de que la temperatura se encuentre fuera de este rango, la señal es todavía dependiente de la temperatura pero la relación entre ambas deja de ser lineal. El dispositivo tiene un sensor que opera en una longitud de onda entre 2 y $2.5 \mu \mathrm{m}$, con la óptica instalada, 650 $\mathrm{mm}$ de distancia focal, el área medida es un círculo de $1.5 \mathrm{~mm}$ de diámetro. La emisividad puede ajustarse entre 0.01 y 0.999 y por lo tanto puede tenerse en cuenta el valor para cualquier superficie. El tiempo de refresco para la señal proporcionada por el detector es de $10 \mathrm{~ms}$, el mismo valor se le asigna al tiempo que invierte el dispositivo en pasar desde el punto medible de temperatura menor hasta el de temperatura mayor. Un diodo láser se utiliza para determinar el punto que está siendo medido, para posicionarlo se dispone de un sistema de tres ejes. El punto escogido en la superficie es el de mayor temperatura sobre la pieza, cuya posición depende de la velocidad relativa entre el haz y la pieza y de el diámetro de haz, su posición se determina con ayuda de la simulación [1]

La señal del detector piroeléctrico, que oscila entre los $450 \mathrm{mV}$ y los $1850 \mathrm{mV}$, está conectado a un sistema de lectura que consiste en una tarjeta de adquisición conectada a un PC. La capacidad de muestreo de la tarjeta de adquisición instalada es de $5 \times 10^{5}$ muestras por segundo, con una resolución de 12 bits, la capacidad de muestreo máxima por canal es entonces la octava parte de la capacidad total. El periodo de muestreo utilizado es de $20 \mathrm{~ms}$, que son suficientes dados los tiempos característicos de los procesos térmicos. Para limitar el ruido, que procede de la fuente de alimentación se utilizó un transformador apantallado y también se usaron cables coaxiales. De este modo el ruido se mantuvo por debajo del $5 \%$.

Una cámara CCD fue utilizada para realizar secuencias de la emisión de luz visible que acompaña al proceso de fusión, cuyo comportamiento puede apreciarse en la figura 2. De la forma y tamaño de la región iluminada se pudo extraer información adicional sobre el proceso.

\section{Modelado del proceso}

Cuando la superficie de nuestra pieza de trabajo es irradiada se induce un campo de temperaturas en la pieza, el objetivo del modelo propuesto es encontrar la solución de este campo de temperaturas y su evolución con el tiempo a partir de las condiciones de contorno inducidas por el movimiento del haz láser sobre la superficie del material teniendo únicamente en cuenta procesos de conducción. El modelo utilizado, como ocurre en otros modelos de tratamiento térmico, tiene las siguientes entradas [2]:

- Distribución de energía del haz.

- Geometría de la probeta que está siendo tratada.

- Propiedades termofísicas, densidad $(\rho)$, capacidad calorífica $\left(C_{p}\right)$ y conductividad térmica $(K)$, dependientes de la temperatura.

Asimismo este modelo incluye como la posibilidad de utilizar coeficientes de absorción diferentes en cada una de las fases, aunque dentro de cada fase estos se suponen constantes. Se considera como coeficiente de absorción la fracción de energía incidente que es absorbida por la pieza. La manera de obtener estos coeficientes de absorción es diferente según se trate de la fase sólida o de la fase fundida, así:

- Fase sólida:

- Se procesa una probeta con unos determinados parámetros de proceso, con ayuda de los sensores se determina el punto en el que ocurre el cambio de fase.

- Manteniendo el diámetro de haz y la velocidad, pero variando la potencia, se busca, con ayuda del programa de simulación, un caso teórico en el que el cambio de fase ocurra en ese mismo instante.

La relación entre la potencia teórica y la potencia experimental nos determina la cantidad que se utilizará como coeficiente de absorción para la fase sólida.

- Fase fundida:

- En una probeta experimental, tratada con unos ciertos parámetros, se determina la profundidad de la región fundida.

- Se busca un caso teórico que tratado con los mismos parámetros de velocidad y diámetro de haz anteriores, produzca la misma profundidad fundida. La relación de potencias entre el caso teórico y el experimental nos proporciona el coeficiente de absorción para la fase fundida.

Determinados los coeficientes de absorción se tienen todos los ingredientes para resolver la ecuación de conducción del calor, se utiliza el Método de los Elementos Finitos con la ayuda del Programa ANSYS®.

TABLA I. COMPOSICIONES (\% EN PESO)

\begin{tabular}{|l|c|c|c|c|c|c|c|c|c|c|c|}
\hline & B & C & Cr & Cu & Fe & Mn & Mo & Ni & P & S & Si \\
\hline AISI 304 & & $<0.08$ & $18-20$ & & $>66$ & $<2$ & & $8-10.5$ & $<0.045$ & $<0.03$ & $<1$ \\
\hline METCO 16C & 4 & 0.5 & 16 & 3 & 2.5 & & 3 & 67 & & & 4 \\
\hline
\end{tabular}
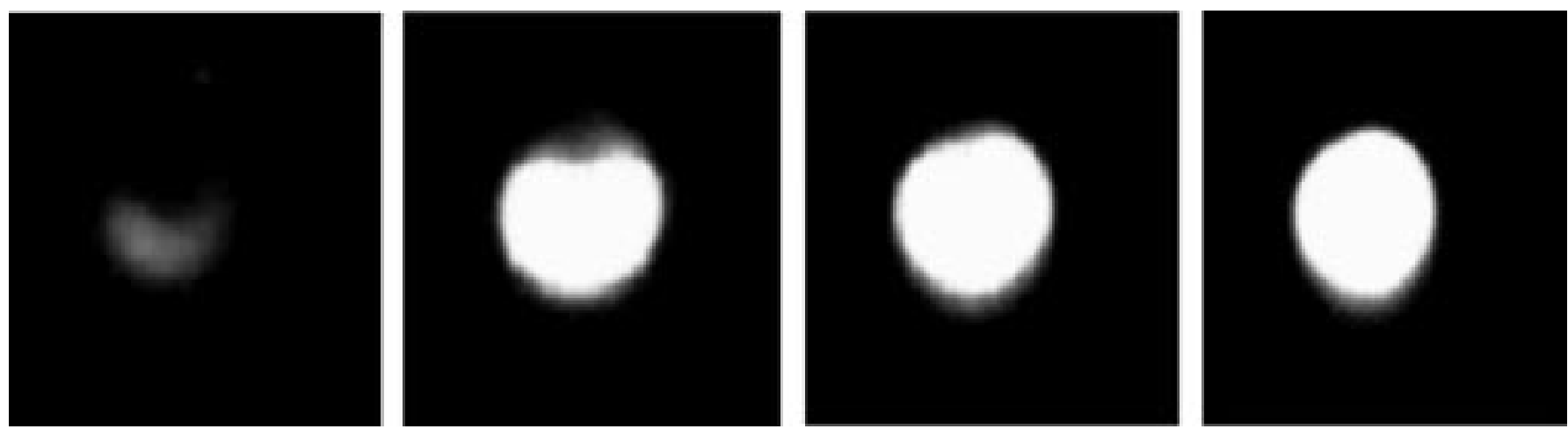

Figura 2. Secuencia registrada con la cámara CCD. Las dos últimas imágenes, de forma prácticamente idéntica se corresponden con el estado estacionario del sistema. 


\section{RESULTADOS}

Se tomaron unas probetas de $50 \times 50 \times 5 \mathrm{~mm}^{3}$ de AISI 304 (Tablas I y II) y se procedió a determinar el coeficiente de absorción para cada una de las fases. En la figura 3 se muestra, con trazo rojo el buen seguimiento que el programa de simulación hace de la evolución de la temperatura en la fase sólida, en total acuerdo con lo determinado con ayuda del pirómetro. Esto permite obtener un coeficiente de absorción en la fase sólida que alcanza el $25 \%$.

TABla II. PropiedAdes TERMOfísicAs DE LOS DOS MATERIALES UTILIZADOS

\begin{tabular}{|c|c|c|c|c|c|c|c|}
\hline \multicolumn{3}{|c|}{ A304 } & \multicolumn{4}{c|}{ METCO16C } \\
\hline $\begin{array}{c}\mathrm{T} \\
{[\mathrm{K}]}\end{array}$ & $\begin{array}{c}\mathrm{K} \\
{[\mathrm{W} / \mathrm{m} / \mathrm{K}]}\end{array}$ & $\begin{array}{c}\mathrm{Cp} \\
{[\mathrm{J} / \mathrm{Kg} / \mathrm{K}]}\end{array}$ & $\begin{array}{c}\rho \\
{\left[\mathrm{Kg} / \mathrm{m}^{3}\right]}\end{array}$ & $\begin{array}{c}\mathrm{T} \\
{[\mathrm{K}]}\end{array}$ & $\begin{array}{c}\mathrm{K} \\
{[\mathrm{W} / \mathrm{m} / \mathrm{K}]}\end{array}$ & $\begin{array}{c}\mathrm{Cp} \\
{[\mathrm{J} / \mathrm{Kg} / \mathrm{K}]}\end{array}$ & $\begin{array}{c}\rho \\
{\left[\mathrm{Kg} / \mathrm{m}^{3}\right]}\end{array}$ \\
\hline 298 & 14.8 & 480 & 8020 & 300 & 11.7 & 472 & 4500 \\
\hline 473 & 17.7 & 530 & 7950 & 455 & 14.4 & 483 & 4436 \\
\hline 673 & 20.7 & 560 & 7855 & 662 & 18.1 & 529 & 4352 \\
\hline 873 & 23.5 & 595 & 7751 & 869 & 21.7 & 583 & 4267 \\
\hline 1073 & 25.8 & 620 & 7645 & 1076 & 25.4 & 640 & 4182 \\
\hline 1273 & 28.8 & 642 & 7532 & $\mathbf{1 2 8 3}$ & 29.0 & 697 & 4097 \\
\hline 1473 & 31.6 & 675 & 7431 & 1460 & 29.0 & 670 & 3651 \\
\hline $\mathbf{1 6 7 3}$ & 33.5 & 730 & 7302 & 1644 & 29.0 & 670 & 3491 \\
\hline 1773 & 29.0 & 800 & 6860 & 1766 & 29.0 & 670 & 3385 \\
\hline 1873 & 30.0 & 800 & 6780 & 1888 & 29.0 & 670 & 3278 \\
\hline
\end{tabular}

Seguidamente y gracias al análisis de esa misma probeta se obtiene la forma de la región fundida que se puede apreciar en la fotografía de la figura 4.a, la profundidad fundida es compatible con un coeficiente de absorción en esa fase del $55 \%$, ese mismo dato permite efectuar la predicción de la forma de la región fundida que se muestra con trazo rojo, si bien esa predicción es mejorable hay que recordar que no se están tomando en consideración los fenómenos de convección, responsables del ensanchamiento de la porción del material que alcanza la fusión. La validación del modelo se realiza extendiendo estos cálculos teóricos a otros conjuntos de parámetros, y de esta manera se obtienen los resultados de la figura 4.b, donde se introducen también las predicciones que no tienen en cuenta el cambio en el coeficiente de absorción.

El mismo procedimiento se realiza con piezas que ya han sido recubiertas por plasma con la aleación base níquel utilizada (METCO 16C) [4] y que son refundidas, en la figura 5.a se aprecia ya el resultado de la predicción de la zona fundida, donde se aprecia que la modelización realizada ofrece mejores resultados dado que predice con gran precisión tanto la anchura como la profundidad de la región tratada.

Como última validación del modelo se ofrece la predicción que este último hace de la evolución temporal de la región fundida en el caso de la refusión de la aleación depositada sobre el AISI 304. Con todas los parámetros ya fijados en los casos anteriores el modelo de simulación es capaz de seguir con gran fidelidad la evolución del tamaño de la región fundida como se aprecia en la figura 5.b.

\section{CONCLUSIONES}

La técnica numérica utilizada, junto con los resultados que vienen de la monitorización en tiempo real del sistema y de la caracterización de los resultados obtenidos permite tener un elevado grado de predicción sobre la forma de las regiones fundidas, aspecto de crucial interés a la hora de realizar procesos de refusión. El modelo permite encontrar los valores adecuados para los parámetros del proceso y

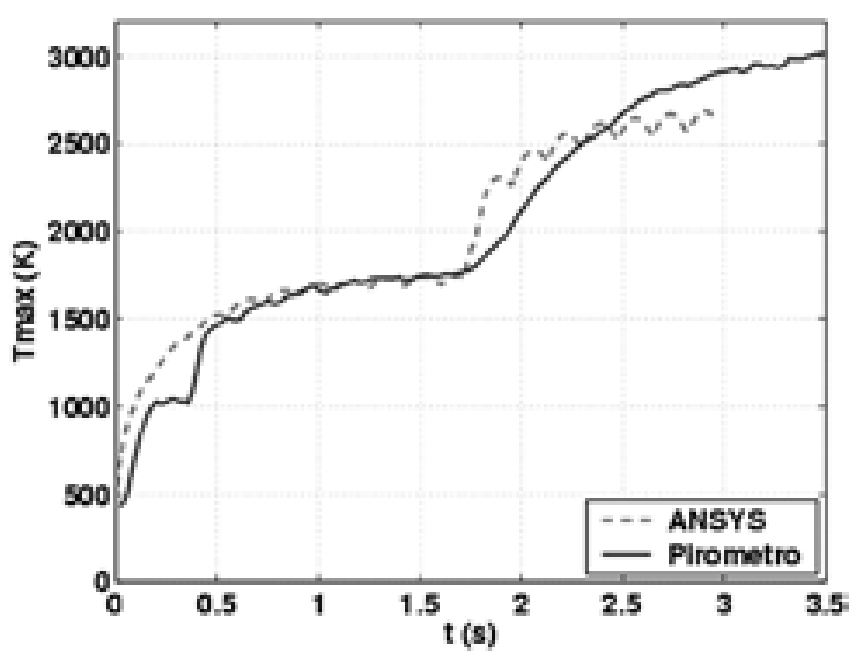

Figura 3. Evolución temporal de la temperatura. Se observa como tiene lugar la transición de fase transcurridos prácticamente $1.8 \mathrm{~s}$.
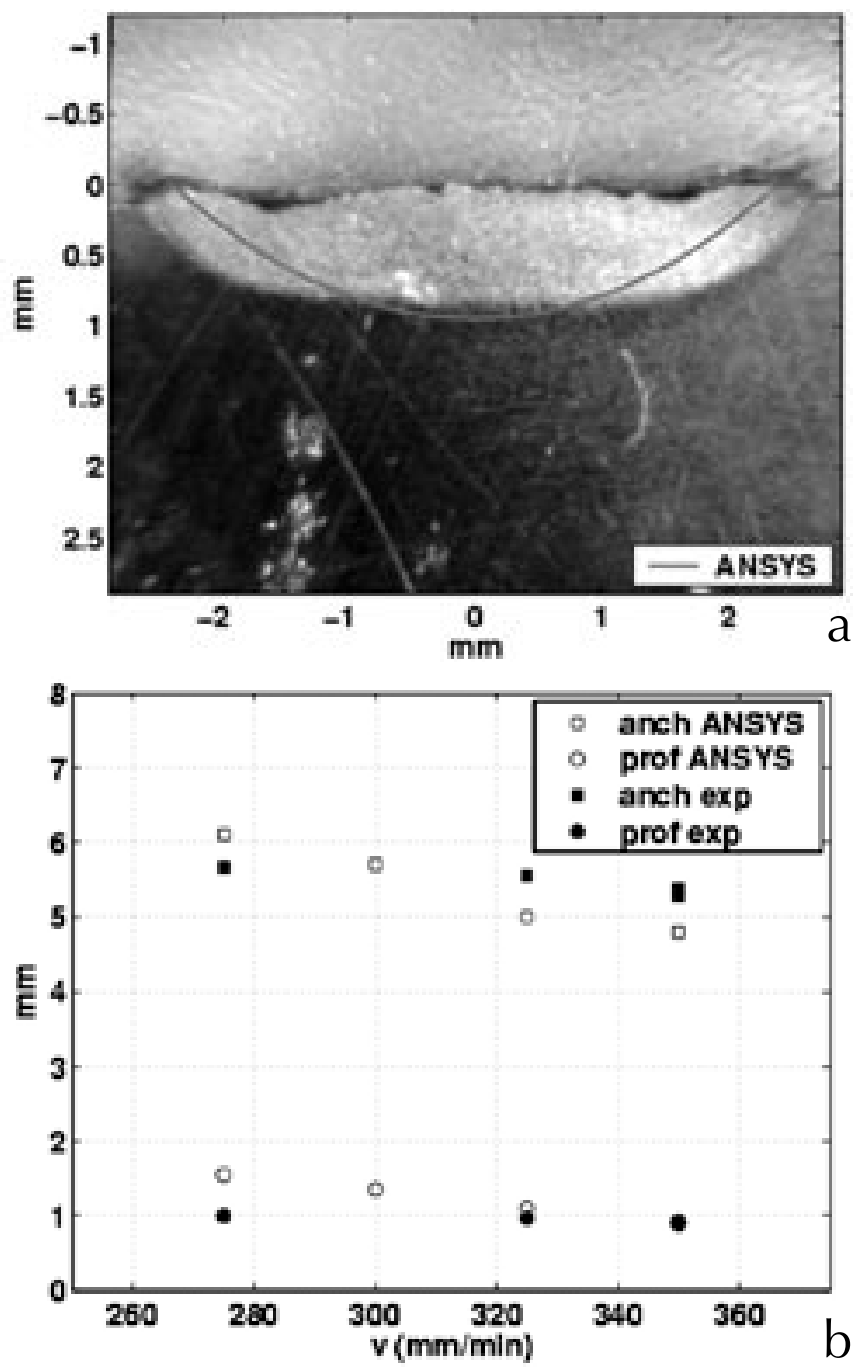

Figura 4. a) Forma de la región fundida según ha sido determinada a partir del análisis de la probeta tratada, se presenta con trazo grueso la predicción realizada por el programa de simulación. b) Extensión del ajuste realizado a otros valores de la velocidad relativa haz-pieza. 

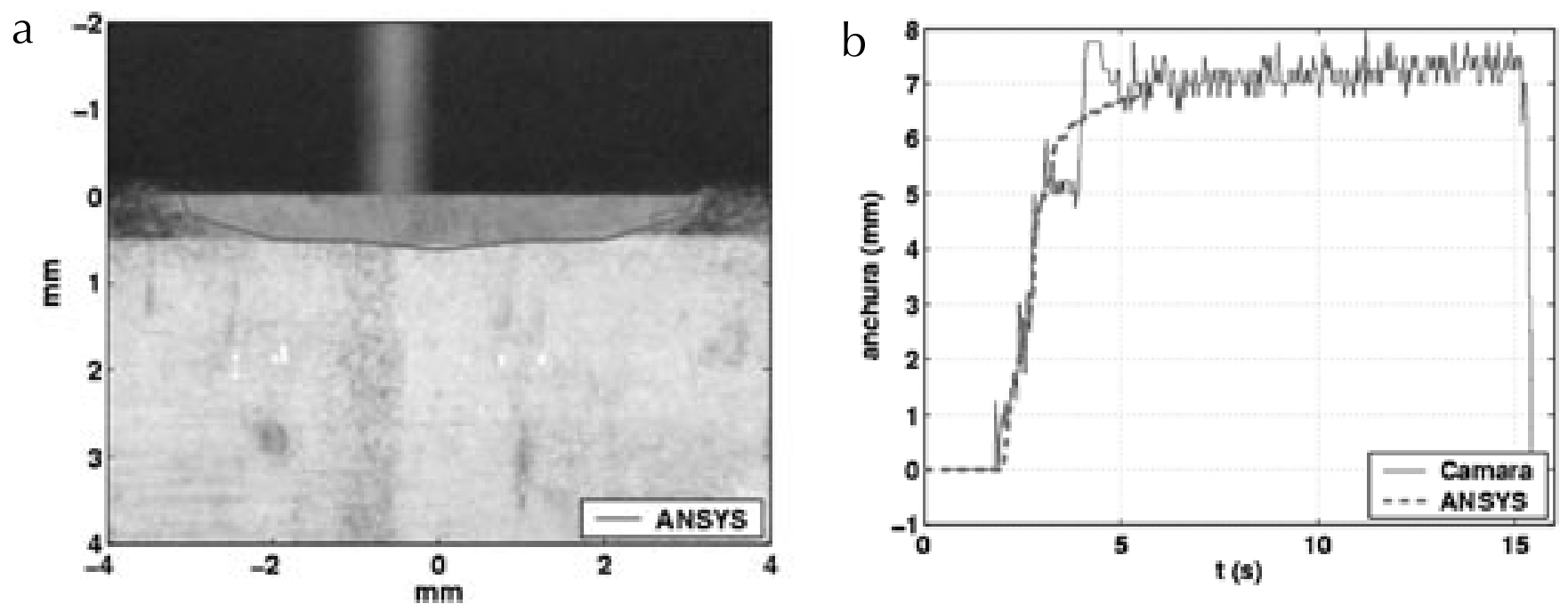

Figura 5. a) Forma de la región fundida en el caso de la refusión de la capa proyectada, se muestra también la predicción. b) Se presenta la evolución temporal de la región fundida, se comparan las imágenes de la cámara CCD con la predicción del modelo.

conocer tanto el mapa de temperaturas como la evolución del mismo. Los parámetros adecuados serán aquellos que consigan fundir simultáneamente toda la capa proyectada y una mínima parte del sustrato lo que asegura una mínima dilución y el consiguiente acercamiento de la composición del recubrimiento a la deseada, que no es otra que la correspondiente al material de aporte.

\section{AGRADECIMIENTOS}

Este trabajo fué realizado gracias a la subvención obtenida del Ministerio de Ciencia y Tecnología y Fondos FEDER (1FD97-2020-C0303), del Programa Ramón y Cajal de ese mismo Ministerio. Los autores quieren agradecer al Centro de Supercomputación de Galicia toda la colaboración prestada en la realización de la parte computacional de este trabajo.

\section{BIBLIOGRAFÍA}

1. C. Alvarez et al.. "Real time control and monitoring of the laser hardening process: application to cylindrical workpieces". Laser Assisted Net Shape Engineering 3. Proceedings. Ed. M. Geiger, A. Otto (2001).

2. A. Yánez et al. "Modelling of temperature evolution on metals during laser hardening process". Appl. Surf. Sci. 186 (2002) 611.

3. K. C. Mills, Recommended values of thermophysical properties for selected commercial alloys. Woodhead Publishing Ltd. ASM International.

4. Metco 16C Self-Fluxing Nickel-Chromium Alloy Powder. Sulzer Metco Technical Bulletin \#10-040.

Recibido: 1.2 .03

Aceptado: 30.11 .03 